\title{
Early sports specialisation and the incidence of lower extremity injuries in youth athletes: current concepts
}

\author{
Richard N Puzzitiello, ${ }^{1}$ Coleen F Rizzo, ${ }^{1}$ Kirsten D Garvey $\odot$, ${ }^{1}$ \\ Elizabeth G Matzkin (1), ${ }^{2}$ Matthew J Salzler (i) ${ }^{1}$
}

${ }^{1}$ Orthopedics, Tufts University School of Medicine, Boston, Massachusetts, USA

${ }^{2}$ Orthopaedic Surgery, Brigham and Women's Hospital, Boston, Massachusetts, USA

\section{Correspondence to}

Dr Matthew J Salzler,

Orthopedics, Tufts University

School of Medicine, Boston, MA

02111, USA:

msalzler@tuftsmedicalcenter.org

Received 15 June 2020 Accepted 15 May 2021 Published Online First 6 July 2021
Check for updates

(C) International Society of Arthroscopy, Knee Surgery and Orthopaedic Sports Medicine 2021. No commercial re-use. See rights and permissions. Published by BMJ.

\section{To cite: Puzzitiello RN} Rizzo CF, Garvey KD, et al. J ISAKOS 2021:6:339-343.

\begin{abstract}
Year-round intensive, single-sport training beginning at an young age is an increasingly common trend in the youth athlete population. Early sport specialisation may be ineffective for long-term athletic success and contribute to an increased risk of physical injury and burn-out. The medical community has noted that repetitive movement patterns may occur in nondiversified activity and this may contribute to overuse injury in young athletes. Studies have begun to identify an association between early sport specialisation and lower extremity injuries in the youth athlete population that is independent of training volume. Recent literature has suggested that sport diversification, not specialisation, is a better path for athletic success and minimised lower extremity injury risk.
\end{abstract}

\section{INTRODUCTION}

The trend towards early sport specialisation plays a role in the estimated 4.5 million sports-related injuries (SRIs) that occur each year to the child and young adult populations. ${ }^{1}$ Between $17 \%$ and $41 \%$ of youth athletes are now considered 'highly specialized' in sports. ${ }^{2}{ }^{3}$ While early sport specialisation may seem advantageous to youth athletes who seek the purported benefits of highly specialised and competitive training, individuals who specialise early are perhaps more susceptible to overuse injuries, lack of cross sport skill development and burn-out. ${ }^{4-6}$ Moreover, it is also unclear whether early specialisation in a single sport can be beneficial for achieving sports mastery. The medical community has cautioned against early sport specialisation to minimise injury risk, while subsequently demanding further research due to the limited data presently available. ${ }^{4-9}$

Early specialisation in a single sport at highintensity and high-volume training may introduce highly repetitive movement patterns and limit the neuromuscular foundational skills that hinder an athlete's long-term development and increase an athlete's risk of overuse injury. ${ }^{10-12}$ Studies suggest that athletes who specialised early were twice as likely to sustain lower extremity injuries (LEIs) than athletes who did not specialise in one sport, and were also more likely to sustain such injuries in organised settings than in free play. ${ }^{13}$ Despite these findings, the effects of early sports specialisation in younger patients ( $<12$ years old) remain largely unexplored.

\section{What is early sport specialisation?}

While there is not an established consensus definition of early sport specialisation, the American

\section{Current concepts}

- Sports specialisation is defined as year-round (8 months or more per year) intensive training in a single sport at the exclusion of other sports.

- Sports specialisation is associated with chronic overuse injuries of the lower extremities even after controlling for sex, age and competition volume.

- Participation in sports for a greater number of hours per week than age in years, participating in a primary sport more than 8 months per year and a ratio of organised sports to free playtime $>2: 1$ are specific risk factors for overuse injuries.

- Multiple organisations denounce early sports specialisation for its ineffective and even dangerous impact on youth athletes.

\section{Future perspectives}

There is a need to assess sports-specific risks and benefits for early specialisation, especially in nuanced technical sports where peak performance occurs prior to full physical maturation

- Identification of injury-specific patterns in specific sports where early specialisation is common. Such data may aid in identifying alternative training methods that may mitigate risk while optimising athletic achievement.

Orthopaedic Society for Sports Medicine (AOSSM) describes early sports specialisation as a commitment to 'year-round (8 or more months per year) intensive training in a single sport at the exclusion of other sports' that began prior to age $12 .{ }^{8}$ Sports specialisation that occurs after the age of 12 is no longer considered 'early sport specialization'. Early specialisation trends indicate that youth athletes are increasingly specialising prior to high school, continuing to play in less than two sports throughout high school and participating in both high school and club teams. ${ }^{3} 1415$ While specialisation and high-volume training may benefit skill development, major drawbacks include the finding that athletes may become more vulnerable to SRIs, overtraining and burn-out as exposure hours increase. $5791316-18$ Athletes who train in one sport year-round at a highly competitive level place added stress on sport-selective groups of muscles and ligaments while reducing the body's development of 
diversified movement patterns that might otherwise increase with multisport participation. ${ }^{10}$

\section{Does sport specialisation increase the incidence of LEls?}

As training volumes intensify, youth athletes become increasingly susceptible to musculoskeletal overuse injuries. ${ }^{19}$ Most notably, injury data reveal an increased number of reported incidences of LEIs of the hip and knee. ${ }^{16}$ As such, these studies may suggest that the repetitive motions and lack of diverse movement patterns associated with overtraining at highly competitive levels may increase the risk of overuse injuries and acute LEIs.

The National Federation of High School Associations funded a large high school athlete population-based analysis study to investigate the association between sport specialisation and the incidence of LEIs. ${ }^{13}$ Level of sport specialisation was categorised using a three-point scale in response to specialisation study questions that included the following: (1) Have you quit another sport to focus on your primary sport? (2) Do you consider your primary sport more important than your other sports? (3) Do you train more than 8 months/year in your primary sport? Participants were then categorised as low (score $=0-1)$, moderate $($ score $=2)$ and high $($ score $=3)$. According to McGuine et al, $21.3 \%$ of high school athletes who qualified as highly specialised sustained an LEI, which was significantly higher than the $12.2 \%$ of those who were low level of specialisation $(p=0.02) .{ }^{13}$ Athletes who classified into the moderate specialisation group also sustained LEIs at a significantly higher rate than low level of specialisation $(18.9 \%, 12.2 \%, \mathrm{p}=0.03)$. When further stratified by acuity of injury, it was found that there was no significant difference in incidence of acute LEIs between levels of specialisation, but moderate and highly specialised athletes were at a significantly greater risk of developing chronic overuse LEIs (high $=10.9 \%$, moderate $=7.4 \%$, low $=2.9 \%$ ). However, this did not result in a significantly greater number of days lost for injuries by the participants across levels of participation, or significantly greater number of surgeries. The results of this study suggest that increasingly specialised athletes are at greater risk of overuse injury even after controlling for sex, age, competition volume and history of LEI.

To further demonstrate the association between sport specialisation and youth athlete injury history, Post et al conducted a population-based survey study of over 2000 youth athletes to determine if athletes who exceed sport volume recommendation are more likely to have history of injury of any type, or chronic overuse injury. ${ }^{20}$ The study found that sport specialisation defined by the same aforementioned three-point scale was an independent risk factor for injury history, which remained true after controlling for age, sex and weekly training volume. ${ }^{20}$ Further, athletes participating in their primary sport for more hours per week than their age, and participating in their primary sport more than 8 months/year, were more likely to report an injury of any kind, and a chronic overuse injury of upper and lower extremities. In another study by Post et al evaluating for associations with only overuse injuries, female high school athletes were more likely to categorise as highly specialised than male high school athletes, and more likely to report previous overuse LEIs than male athletes in sex-equivalent sports. ${ }^{21}$ Overall, athletes who exceeded volume recommendations or played a single sport were more likely to report a history of LEIs, ${ }^{22}$ which may be influenced by the trend towards earlier sport specialisation.

In a case-control study by Jayanthi et al, 882 injured patients from a sports medicine clinic were compared with 398 uninjured healthy controls. ${ }^{22}$ These athletes were categorised by degree of sports specialisation using the same three-point scale used in the study by McGuine et al. After controlling for baseline variables, sports specialisation was found to be an independent risk factor for chronic overuse injury, and serious overuse injury as defined as an injury requiring at least 1-month rest from sports. However, highly specialised athletes were not at a greater risk for acute injuries. They also found that athletes participating in sports for a more number of hours per week than their age in years, and those whose ratio of organised sports to free playtime was $>2: 1$, were also at significantly increased risk of serious overuse injury. This study demonstrated that sports specialisation alone, independent of age and training volume, significantly increased the risk of overall and serious overuse injury.

The aforementioned studies by Jayanthi et al, Post et al and McGuine et al include high school athletes mostly over the age of 12. In contrast, the cross-sectional survey study by McGowan et al included only athletes $<13$ years old, thus specifically investigating the effects of early sports specialisation. ${ }^{23}$ This study used a slight modification of the three-point scale used in the previously mentioned studies to categorise degree of sports specialisation. They found that after adjusting for age, sex and volume of sports participation, injury was not significantly associated with early sports specialisation. However, exceeding the recommended sport participation volumes (more hours of sport per week than age in years, playing one sport for more than 8 months of the year and exceeding a 2:1 weekly ratio of organised sport to recreational free play hours) was associated with increased odds of chronic overuse injuries. These results are contrary to the previously mentioned studies. The authors suggest this difference in findings may be due to the higher relative amount of 'moderately specialized' athletes in their population who participate in a diversified secondary sport. Additionally, these younger specialised athletes may benefit from improved access to resources and informed coaching which may improve their technique and physical condition in an early specialised learning environment. Finally, these younger athletes have fewer years of sports specialisation compared with the previously discussed studies. Thus, it is possible that risk of injury may in fact be associated with early sports specialisation if maintained over several years into adolescents.

These studies collectively demonstrate that specialised athletes are at an increased risk for chronic overuse injury, but are not at an increased risk for acute injuries when a delineation in injury type is made. However, these studies by Jayanthi et al, Post et al and McGuine et al include high school athletes mostly over the age of 12, and thus are not specific to early sports specialisation. In contrast, the study by McGowan et al suggests early sports specialisation is not a risk factor for overuse injuries nor injuries of any type, but exceeding volume of training recommendations is. Further investigation is needed to explore whether early sports specialisation may portend an increased risk for injury. More specifically, prospective studies investigating the risk of sports specialisation from prior to growth spurts to past the time of skeletal maturity would provide significant insight on the variations in associated risk during times of peak musculoskeletal growth.

\section{Sex and sports-specific differences in sports specialisation and overuse injuries}

Several recent studies have also explored the sex-based differences of sports specialisation and risk of chronic overuse injuries. $^{24-26}$ On further stratification by injury type, they found that specialised and non-specialised had similar odds of developing 
a chronic overuse LEI, but non-specialised athletes were more likely to report upper extremity and trunk overuse injuries. Post et al recently published a questionnaire-based cross-sectional study on soccer, basketball and volleyball athletes aged 12-18 years, which demonstrated that female basketball athletes were four times more likely than male basketball athletes to report an overuse injury, controlling for level of specialisation and volume of training. ${ }^{25}$ Furthermore, high levels of specialisation were significantly associated with overuse injuries among volleyball athletes, which was one of the most popular sports for female athletes. The same authors published an additional study on adolescent athletes aged 12-18 years, who participated in volleyball, baseball, soccer, softball, basketball, track and field, lacrosse, swimming or ice hockey. They found in this study that highly specialised athletes were more likely to report both acute and overuse injuries compared with low specialisation athletes. However, when stratified by sex, only specialised females were more likely to report an overuse injury. This association was maintained after controlling for age and volume of sports participation. Sports specialisation in male athletes was not associated with overuse or acute injuries. ${ }^{27}$

In the epidemiological study by Schroeder et al, 2834 overuse injuries were evaluated and female athletes were found to have sustained overuse injuries at a greater rate compared with their male counterparts $(2.08$ vs 1.44$){ }^{11}$ These injuries were most frequently observed in the lower extremity. The highest rates of overuse injury were observed in females participating in lacrosse, field hockey and track and field. ${ }^{11}$ Sports that required repetitive movements of the lower body, such as track and field and soccer, reported the highest rates of lower leg injuries. ${ }^{11}$ Sex patterns became distinct when analysing the proportions of overuse injuries distributed across the 4 years of high school. ${ }^{11}$ In males, overuse injuries increased from freshman through senior year (20.9\%-28.5\%), while the proportion of overuse injuries in females decreased from $30 \%$ as freshmen to $19.8 \%$ as seniors. ${ }^{11}$ They explained this finding by offering that females were more likely to sustain overuse injuries at earlier ages than males due to factors including an earlier age of sexual maturity and different biomechanics than males. ${ }^{11}$ This study did not however analyse the impact which sports specialisation has on these results.

These findings may indicate that females are more susceptible to LEI overuse injuries and that there are sports-specific patterns of LEIs which may be explained by sport-specific movements (ie, cutting, pivoting). The prevalence of lower extremity overuse injuries in younger female athletes creates room for investigation into the sex-related impact of repetitive, non-diversified patterns of single sport specialisation.

\section{Current recommendations}

Professionals have grown fearful of the demands of specialisation on youth athletes, leading to the release of position statements from medical societies concerning the growing practice of early specialisation and campaigns.

The OneSport Injury campaign was created by the AOSSM and the American Academy of Orthopaedic Surgeons to help raise awareness among parents, athletes and coaches of the risk of overuse sports injuries that come with sports specialisation and safe practices to avoid such injuries. These recommendations are mostly level $\mathrm{V}$ evidence in themselves, and advocate for playing different positions or different sports throughout the year, taking one season off each year, and to not play while injured. ${ }^{4-9}$ The American Academy of Pediatrics (AAP) released recommendation guidelines in 2015 purposed to assist paediatricians in counselling their young athletic patients and parents regarding sports specialisation and intensive training. These guidelines emphasise that the focus of sports should be to have fun and learn lifelong physical activity skills. Additionally, the AAP suggests a higher chance of reaching athletic goals if you have early diversification of sport until puberty, specialisation not until late adolescence, taking at least three 1 -month periods of rest from primary sport and taking 1-2 days off per week from primary sport. ${ }^{4}$ Similarly, the AOSSM and the American Medical Society for Sports Medicine (AMSSM) state that sport specialisation may be disadvantageous for youth athletes and advocate for multisport participation, non-competitive play and prevention efforts to minimise injury risk. ${ }^{78}$ Both the International Olympic Committee and the AMSSM promote diversification in most sports for the reduction of injury risk and promotion of sport enjoyment over competition. ${ }^{7}$ The messages from these medical societies present a unified, although not entirely evidence-based front, which encourages youth athletes to participate in more than one sport to reduce the potential risk of injury that can result from specialisation.

\section{DISCUSSION}

Current literature reveals the medical community's concern that sport specialisation increases the risk of chronic overuse injuries, especially in the lower extremity. ${ }^{12}$ Recent studies have consistently demonstrated that sports specialisation is associated with chronic overuse injuries of the lower extremities even after controlling for sex, age and competition volume. However, further research is needed to elucidate whether the same risks are associated with early sports specialisation in athletes $<12$ years old. Additionally, evidence suggests that females are more susceptible to LEI overuse injuries and that there are sports-specific patterns of LEIs which may be explained by sport-specific movements (ie, cutting, pivoting). Overall, the scientific community denounces sports specialisation for its ineffective and even dangerous impact on young athletes, citing the link between sports specialisation and increased incidence of overuse injury and burn-out. ${ }^{4-9}$ The authors of this article have the following evidence-based recommendations from the findings of the current review: specialised female athletes should be cautioned of their heightened risk of overuse injuries, athletes should participate in their primary sport less hours per week than their age, athletes should participate in their primary sport no more than 8 months/year and athletes should not exceed a ratio of 2:1 for time spent a week on organised sports to free playtime.

It is commonly believed in sports communities that sports specialisation at a young age will in general lead to greater athletic achievement. The '10000 hours' theory for achieving expertise in a field is often quoted as being applicable for sports training. ${ }^{23}$ However, this popular rule was initially proposed for musicians, and has never been proven to be true for athletes. Athletes who are submitted to such rigorous training at a young age are at risk of social isolation from peers, altered relationships with family and maladaptive behaviours. ${ }^{627} 28$ It is also unclear whether early specialisation in a single sport can be beneficial for achieving sports mastery. Several large studies evaluating elite athletes have demonstrated that these individuals were more likely to have participated in multiple sports, and less likely to have specialised in their respective sport at a younger age. ${ }^{29} 30$ Despite this evidence, large prospective studies are needed to better determine if there may be benefits for early sports specialisation. 
Recognising the established concern for chronic overuse LEI risk, the medical community supports youth athlete diversification of sport through a long-term athlete development model in order to prioritise sport enjoyment and cross development of movement patterns. ${ }^{4-9}$ One set of guidelines recommends that youth athletes aged 15-16 years are at the safest age to consider specialisation in sport; however, the exact amount of training needed for sport success or a threshold for avoidance of such injuries remains unknown. ${ }^{4}$ That is not to say that sports diversification is the only option for youth athletes, especially since some athletes may enjoy playing one sport above others, at an early age. If an athlete does prefer a single sport, the risk of overuse injury may be mitigated if specialisation occurs in a rewarding and sustainable environment that they find enjoyable. ${ }^{24}$ In any case, the choice to specialise should be given to the athlete without the external pressures from parents, coaches and society, provided the athlete is given the information on the risks of sport specialisation, in addition to strategies and training guidelines to avoid injury risk. ${ }^{1-3}$ Effective strategies and prevention measures include sex-specific weightlifting programmes designed for optimal athletic performance and resistance to injury, nutritional counselling and ensuring adequate recovery periods. ${ }^{4}$

Despite the evidence presented in this review, there is still little evidence regarding the injuries associated with early sports specialisation. Future studies are warranted to further elucidate the risks and benefits of early specialisation, especially in nuanced technical sports where peak performance occurs prior to full physical maturation. For instance, sports such as golf, gymnastics and figure skating are not dependent on physical growth and maturation and may even be detrimental in some instances. ${ }^{11}$ Therefore, specialisation in these sports may seem intuitive and naturally beneficial to athletes, coaches and parents. Future studies are also necessary for identification of injury-specific patterns in specific sports where early specialisation is common. Such data may aid in identifying alternative training methods that may mitigate risk while optimising athletic achievement. Finally, much of the discussed literature on the topic of sports specialisation and susceptibility to injury pertains to adolescent athletes, which does not fit the AOSSM definition for early sports specialisation. As such, future studies are needed to identify further associations between early sports specialisation and injuries in younger age groups.

\section{CONCLUSION}

Overall, the medical community recognises the minimal benefits and the numerous drawbacks of adolescent and youth sports specialisation. The major concern regarding sports specialisation at a young age is that specialisation is unnecessary for athletic success, and is physically and mentally harmful. Current literature presents a uniform opinion that athletes who specialise in early adolescence are at a greater risk for LEI. Guidelines surrounding the proper timing of youth athlete specialisation and sex-specific injury prevention measures should be implemented to keep youth athletes active and healthy. While multisport participation may be one answer to the increased injury risk due to early specialisation, specialised athletes may also be capable of minimising injury risk given the proper tools and training.

Twitter Elizabeth G Matzkin @DocMatzkin

Contributors RNP substantially contributed to drafting and revising the work fo important intellectual content, and to conception and design and final approval of the version to be published. CFR and KDG substantially contributed to conception and design of the work as well as drafting and revising the work. EGM and MJS substantially contributed to conception and design as well as to final approval of the version to be published.

Funding The authors have not declared a specific grant for this research from any funding agency in the public, commercial or not-for-profit sectors.

Competing interests None declared.

Patient consent for publication Not required.

Provenance and peer review Commissioned; externally peer reviewed.

\section{ORCID iDs}

Kirsten D Garvey http://orcid.org/0000-0002-2507-0537

Elizabeth G Matzkin http://orcid.org/0000-0001-8592-7187

Matthew J Salzler http://orcid.org/0000-0002-9928-0879

\section{REFERENCES}

1 Fernandez WG, Yard EE, Comstock RD. Epidemiology of lower extremity injuries among U.S. high school athletes. Acad Emerg Med 2007;14:641-5.

2 Post EG, Thein-Nissenbaum JM, Stiffler MR, et al. High school sport specialization patterns of current division I athletes. Sports Health 2017;9:148-53.

3 Bell DR, Post EG, Trigsted SM, et al. Sport specialization characteristics between rural and suburban high school athletes. Orthop J Sports Med 2018;6:232596711775138.

4 Brenner JS, Council on Sports Medicine and Fitness. Sports specialization and intensive training in young athletes. Pediatrics 2016;138:e20162148. doi:10.1542/ peds.2016-2148

5 Jayanthi N, Pinkham C, Dugas L, et al. Sports specialization in young athletes: evidence-based recommendations. Sports Health 2013;5:251-7.

6 Malina RM. Early sport specialization: roots, effectiveness, risks. Curr Sports Med Rep 2010;9:364-71.

7 DiFiori JP, Benjamin HJ, Brenner JS, et al. Overuse injuries and burnout in youth sports: a position statement from the American medical Society for sports medicine. $\mathrm{Br} J$ Sports Med 2014;48:287-8.

8 LaPrade RF, Agel J, Baker J, et al. AOSSM early sport specialization consensus statement. Orthop J Sports Med 2016:4:232596711664424

9 OneSport overuse injury. Available: https://www.orthoinfo.org/onesportinjury [Accessed 15 Feb 2020].

10 Myer GD, Jayanthi N, Difiori JP, et al. Sport specialization, part I. Sports Health 2015;7:437-42.

11 Schroeder AN, Comstock RD, Collins CL, et al. Epidemiology of overuse injuries among high-school athletes in the United States. J Pediatr 2015;166:600-6.

12 Patel DR, Yamasaki A, Brown K. Epidemiology of sports-related musculoskeletal injuries in young athletes in United States. Trans/ Pediatr 2017;6:160-6.

13 McGuine TA, Post EG, Hetzel SJ, et al. A prospective study on the effect of sport specialization on lower extremity injury rates in high school athletes. Am J Sports Med 2017:45:2706-12.

14 Buckley PS, Bishop M, Kane P, et al. Early Single-Sport specialization: a survey of 3090 high school, collegiate, and professional athletes. Orthop J Sports Med 2017:5:232596711770394. doi:10.1177/2325967117703944

15 Post EG, Bell DR, Trigsted SM, et al. Association of competition volume, Club sports, and sport specialization with sex and lower extremity injury history in high school athletes. Sports Health 2017;9:518-23.

16 O'Kane JW, Neradilek M, Polissar N, et al. Risk factors for lower extremity overuse injuries in female youth soccer players. Orthop J Sports Med 2017:5:2325967117733963

17 Wiersma LD. Risks and benefits of youth sport specialization: perspectives and recommendations. Pediatr Exerc Sci 2000;12:13-22. doi:10.1123/pes.12.1.13

18 Bridge MW, Toms MR. The specialising or sampling debate: a retrospective analysis of adolescent sports participation in the UK. J Sports Sci 2013;31:87-96.

19 Brenner JS, Small EW, Bernhardt DT, American Academy of Pediatrics Council on Sports Medicine and Fitness. Overuse injuries, overtraining, and burnout in child and adolescent athletes. Pediatrics 2007:119:1242-5.

20 Post EG, Trigsted SM, Riekena JW, et al. The association of sport specialization and training volume with injury history in youth athletes. Am J Sports Med 2017:45:1405-12.

21 Brooks MA, Post EG, Trigsted SM, et al. Knowledge, attitudes, and beliefs of youth Club athletes toward sport specialization and sport participation. Orthop J Sports Med 2018;6:232596711876983.

22 Jayanthi NA, LaBella CR, Fischer D, et al. Sports-specialized intensive training and the risk of injury in young athletes: a clinical case-control study. Am J Sports Med 2015:43:794-801.

23 McGowan J, Whatman C, Walters S. The associations of early specialisation and sport volume with musculoskeletal injury in New Zealand children. J Sci Med Sport 2020;23:139-44

24 Frome D, Rychlik K, Fokas J, et al. Sports specialization is not associated with greater odds of previous injury in elite male youth soccer players. Clin J Sport Med 2019;29:368-73 
25 Post EG, Biese KM, Schaefer DA, et al. Sport-Specific associations of specialization and sex with overuse injury in youth athletes. Sports Health 2020;12:36-42.

26 Hall R, Barber Foss K, Hewett TE, et al. Sport specialization's association with an increased risk of developing anterior knee pain in adolescent female athletes. I Sport Rehabil 2015;24:31-5.

27 Biese KM, Post EG, Schaefer DA, et al. Evaluation of adolescent sport specialization and injury mechanism by sex: a secondary analysis. J Sci Med Sport 2020;23:721-5.
28 Malina RM. Children and adolescents in the sport culture: the overwhelming majority to the select few. J Exerc Sci Fit 2009;7:S1-10.

29 Moesch K, Elbe A-M, Hauge M-LT, et al. Late specialization: the key to success in centimeters, Grams, or seconds (CGS) sports. Scand I Med Sci Sports 2011;21:e282-90.

30 Güllich A, Emrich E. Evaluation of the support of young athletes in the elite sports system. Eur J Sport Soc 2006;3:85-108. 